\title{
Yod
}

Revue des études hébraïques et juives

La littérature israélienne, miroir d'une société multiple

\section{L'univers domestique et la banalité quotidienne chez les romancières israéliennes}

Everyday Life and Domestic World in Israeli Contemporary Women's Fiction

$$
\text { חיי היום יום בספרות הנשים הישראלית }
$$

\section{Ziva Avran}

\section{(2) OpenEdition}

\section{Journals}

Édition électronique

URL : https://journals.openedition.org/yod/383

DOI : $10.4000 /$ yod.383

ISSN : 2261-0200

Éditeur

INALCO

Édition imprimée

Date de publication : 1 octobre 2009

Pagination : 215-231

ISBN : 978-2-85831-178-1

ISSN : 0338-9316

\section{Référence électronique}

Ziva Avran, «L'univers domestique et la banalité quotidienne chez les romancières israéliennes », Yod [En ligne], 14 | 2009, mis en ligne le 01 novembre 2011, consulté le 08 juillet 2021. URL : http:// journals.openedition.org/yod/383 ; DOI : https://doi.org/10.4000/yod.383

Ce document a été généré automatiquement le 8 juillet 2021.

\section{(c) (1) (9)}

Yod est mis à disposition selon les termes de la Licence Creative Commons Attribution - Pas d'Utilisation Commerciale 4.0 International. 


\title{
L'univers domestique et la banalité quotidienne chez les romancières israéliennes
}

\author{
Everyday Life and Domestic World in Israeli Contemporary Women's Fiction \\ חיי היום יום בספרות הנשים הישראלית
}

\section{Ziva Avran}

1 Dans son livre La Voix d'une femme juive ${ }^{1}$, Tova Cohen évoque la fonction du quotidien dans le roman féminin du XIX siècle. Son analyse, inspirée des recherches sur l'expression féminine à l'époque victorienne et appliquée aux premières écrivaines de la Haskala, met en avant le vécu féminin et ses particularités dans la description de certains travaux ménagers, notamment dans les scènes de cuisine.

2 Cette tentative de recherche identitaire à travers un sujet déconsidéré par les féministes du XXe siècle m'a poussée à m'interroger sur la présence ou l'absence de cette thématique chez les romancières israéliennes contemporaines. Quelle est la place de l'univers domestique dans cette production romanesque et quelle en est la fonction? Comment s'intègre-t-il dans la narration et quel est son impact?

3 Les œuvres analysées dans le cadre de cette recherche sont composées pour la plupart à partir des années 90 , marquées par une présence de plus en plus patente des femmes sur la scène littéraire israélienne. Ce choix implique essentiellement une vision synchronique qui n'exclut cependant pas quelques écarts, notamment dans les références à Amalia Kahana-Carmon, figure incontournable de cette prise de conscience, clairement exprimée dans sa fiction comme dans ses $\operatorname{articles}^{2}$ et ses nombreuses interventions orales.

4 Une lecture ciblée de ce corpus fait ressortir deux attitudes contradictoires dans l'expression identitaire du quotidien: d'une part le désir de raconter le vécu féminin étroitement lié à l'univers domestique, d'autre part la volonté de l'écarter, en s'attaquant notamment à l'extrême idéalisation de la cellule familiale. Parallèlement à l'extension de ces expériences particulières ${ }^{3}$ qui évoluent vers des régions plus intimes 
(maternité, sexualité, etc.), on assiste également à l'émergence d'une prose revendicatrice. Dans la filiation directe des idées féministes, cette prose désigne la sphère familiale et avec elle la banalité quotidienne comme source d'inégalités, véritable entrave au bonheur et à l'épanouissement de la femme.

5 Conformément à l'orientation idéologique choisie par la romancière, l'univers domestique deviendra synonyme de banalité et de piège ou apparaîtra comme un simple vécu, exempt de tout jugement de valeur. Matériau romanesque ou simple miroir, cette réalité quotidienne influera inévitablement sur la poétique du récit, conditionnant également certains choix stylistiques.

\section{L'univers domestique ou les pièges de la banalité quotidienne}

\section{Amalia Kahana-Carmon}

6 Amalia Kahana-Carmon, sans doute, la plus féministe des écrivaines israéliennes, qui a mené une véritable réflexion sur la place de la femme et son statut d'artiste dans une société marquée par les traditions patriarcales, n'a cessé de dénoncer les pièges de la banalité quotidienne. Dans Et la lune dans la vallée d'Ayalon ${ }^{4}$, la femme mariée est perdante qu'elle soit dominée ou dominatrice, toujours accablée par l'obligation de gérer la « machine du foyer », une obligation qu'elle qualifie de désastre (p.151) 5 .

7 Synonyme de grisaille, l'univers domestique n'inspire que lassitude et désespoir, naissant toujours d'un contraste entre le rêve et la réalitée. "Tout était nouveau ", constate la jeune fille qui passe brusquement du bal de fin d'année à une existence besogneuse, «faire bouillir de l'eau, mais comment exactement. Se laver les mains et la figure, mais comment exactement. Laver les chaussettes et les mettre à sécher, mais comment exactement. Brosser les bords de la robe de bal, les chaussures, gratter la boue, mais comment exactement. Ranger, nettoyer. Coudre, rapiécer. » (« Après le bal de fin d'année $»^{7}$, p. 249).

8 Les héroïnes d'Amalia Kahana-Carmon sont les victimes du quotidien, dans leur propre vision, mais parfois aussi dans celle de leurs enfants dont le regard clairvoyant perçoit l'étendue du malaise. Une jeune fille qui vit sa première expérience amoureuse, observe sa mère épuisée par les tâches domestiques, bien décidée à échapper au même destin ("Voile»). Un garçon s'apitoie sur sa mère tiraillée entre ses rêves d'amour et ses obligations familiales, conscient de son échec et de ses humiliations ( «Au coeur de l'été, au coeur de la lumière»)"

\section{Et ses héritières...}

9 Aussi surprenant que cela puisse paraître, Amalia Kahan-Carmon n'aura pas de véritables héritières pour porter d'une manière aussi directe, à la fois tragique et revendicatrice, le flambeau du féminisme prônant la destruction des mythes et la dévalorisation de la sphère familiale. Dédramatisée, la contestation est souvent teintée d'humour et d'ironie. La prose composée par des femmes dans les dernières décennies semble adoucir le discours pour mieux exposer leurs revendications. 
10 En dehors de quelques manifestations épisodiques ${ }^{9}$, la véritable remise en question du système patriarcal, tant dans ses idées que dans ses implications quotidiennes, apparaît dans la prose de Orly Castel-Bloom dont la démarche subversive ébranle les idées reçues les plus ancrées dans les mentalités. De toute évidence, le post-modernisme, avec sa décomposition systématique des formes et des contenus, sied parfaitement à cette nouvelle étape de contestation. Le démantèlement de l'intrigue, la déstructuration des personnages dans le sens traditionnel du terme et l'esprit subversif qui tourne en dérision l'existence même d'une approche idéologique en affirmant parfois une chose et son contraire permettent à l'auteur d'aborder d'une manière désinvolte les revendications chères aux féministes avec une véritable interrogation sur des notions sacralisées telles que l'amour filial ou le sentiment maternel. Orly Castel-Bloom poursuit ainsi, d'un roman à l'autre, son questionnement sur la place de la femme qui cherche à concilier l'inconciliable et à réussir dans ses multiples fonctions : mère, épouse, maîtresse, artiste, etc. Abordée souvent avec d'autres thèmes, la bataille féministe (qui ne se définit jamais comme telle) constitue le principal sujet de La Mina Lisa ${ }^{10}$, une fable dont l'héroïne est une femme au foyer heureuse de l'être :

Je suis une femme au foyer, j'avais envie de prendre la voiture et d'arriver dans une autre maison, une maison dans la vallée, pour y être une femme au foyer, commencer à épousseter, examiner la situation des placards de cuisine, couvrir peut-être des étagères de papier. ( $\mathrm{p}$.

86)

11 La narratrice énumère consciencieusement, sans jamais se plaindre, ses obligations de mère de famille et ses travaux domestiques : accompagner les enfants à leurs diverses activités, charger le lave-vaisselle avec les munitions adéquates, nettoyer la table et ce qui tombe dessous, ramper et ramasser ce qui s'y trouve, laver le carrelage, mettre les casseroles encore tièdes dans le réfrigérateur, s'agenouiller et faire rangement dans le frigo, etc. (pp. 50, 51)

12 Dans un discours faussement innocent, l'héroïne ne se contente pas d'exécuter naturellement ces tâches, mais intériorise aussi l'obligation dictée par les conventions (p. 20) ainsi que le jugement dévalorisant et machiste porté pas son mari sur l'ensemble de ses activités (pp.38-39). Le récit étant livré à la première personne, la critique s'exprime par l'ironie, à la fois verbale et pragmatique. L'inadéquation entre le discours et la situation est d'autant plus grande, que l'auteur multiplie ${ }^{11}$ les affirmations tendant à prouver le bien-fondé de cette condition féminine, faisant ressortir l'ironie par l'accumulation de modalisateurs verbaux et l'emphase :

Mes enfants revinrent de l'école, je les conduisis à leurs activités diverses, j'attendis qu'ils eussent fini, je fis ce qui m'incombait, comme c'est écrit dans les livres, comme on le montre dans les films, comme chacun sait qu'il faut le faire [...] (p. 20)

13 Dans d'autres romans, le refus de cette banalité quotidienne s'exprime justement par son absence. Dans Dolly City ${ }^{12}$, où le sentiment maternel du personnage se manifeste principalement dans les excès d'angoisse, les rares gestes quotidiens s'inscrivent toujours dans une démence frénétique. En mettant en marche le lave-linge, la mère affolée imagine son bébé, avec le linge, à l'intérieur du tambour et perd aussitôt connaissance (p. 23). Quant à la cuisine, Dolly ne confectionne qu'un seul plat : elle fait griller son poisson rouge après l'avoir découpé en lamelles. (p. 14)

14 L'intrigue rocambolesque de Où je suis ${ }^{13}$ exclut toute description d'une vie bien rangée tant sur le plan professionnel que familial. Les rares tentatives culinaires de l'héroïne se soldent par un échec. Une description relativement longue et minutieuse de la 
fabrication des gâteaux, qui reproduit fidèlement les instructions précises d'une recette, accentue la disproportion ironique entre le travail fourni et le résultat :

J'étais dans la cuisine du nouvel appartement et je pétrissais la pâte. Je pétrissais et pétrissais, j'ajoutais un peu de farine et pétrissais encore. J'ai pris un rouleau et j'ai étalé. Je voulais faire des petits gâteaux, je voulais les réussir comme toutes les réussissent. J'ai pris un verre et j'ai découpé des formes rondes. J'ai huilé et légèrement fariné le fond d'un plat carré.

Un rond et encore un rond et encore un rond. J'ai ouvert le four préchauffé, il était brûlant. J'ai enfourné ma pâtisserie et j'ai allumé une cigarette [...].

Les petits gâteaux ont monté, ils ont brûlé sur les bords. J'en ai retiré cinq ou six qui paraissaient bons et je les ai mangés. Je les ai trempés dans ma tasse de café. Ils étaient salés. (pp. 97-98)

Dans une succession de brefs chapitres dignes d'un roman picaresque, l'auteur conçoit une série d'épisodes improbables, entraînant son personnage impétueux et impatient loin de l'appartement conjugal. Le dénouement matérialise la rupture définitive avec le quotidien, aussi confortable soit-il, car la jeune femme quitte le quartier résidentiel de Neot Afeka pour trottiner vers la lune (p. 218).

\section{L'univers domestique ou le quotidien exempt de banalité}

\section{Le « poème domestique »}

Une autre prose, conjuguée au féminin, offre aux lecteurs une vision plus conciliante de l'univers domestique où le quotidien ne rime plus avec banalité. Loin de la contestation ironique de Orly Castel-Bloom, débarrassée des accents dramatiques que lui confère Amalia Kahana-Carmon, la vie au jour le jour, racontée par des écrivaines telles que Zeruya Shalev, Shifra Horn, Mira Magen, Yaël Hedaya ou Tamar Gelbetz est inséparable d'un vécu féminin inhérent à l'intrigue ${ }^{14}$. Au-delà des particularités propres à chacun de leurs romans, on esquisse aisément une série de similitudes avec la mise en place de personnages ayant le même profil, vivant des situations parfaitement analogues. Mère célibataire, divorcée ou en voie de séparation, par choix ou par contrainte, parfaitement intégrée dans la vie active, l'héroïne de ces romans qui vit une histoire d'amour ou de désamour, partage aussi son quotidien avec un enfant. L'univers domestique se constitue donc autour de cette relation où la réitération de mêmes gestes n'est jamais vécue comme une activité ennuyeuse, mais plutôt comme un rituel accompli par les protagonistes ${ }^{15}$.

Intrigues similaires, situations analogues, personnages conçus d'après le même profil autant d'éléments qui contribuent à la constitution de récits proches aussi bien dans leur déroulement que dans leurs détails quotidiens. En racontant l'histoire d'une rupture ou la naissance d'une nouvelle aventure amoureuse, ces écrivaines retracent les mêmes parcours qui se déroulent selon le même schéma et débouchent souvent sur un dénouement heureux ${ }^{16}$. En évoquant la relation mère/enfant, elles décrivent des scènes quasiment identiques. Au-delà d'une fonction romanesque que peuvent avoir ces scènes dans la composition de l'intrigue, leur évocation témoigne aussi d'une simple volonté de raconter un vécu quotidien où l'enfant est omniprésent. La narration consigne minutieusement l'éveil et le coucher de l'enfant, le brossage des dents, le 
passage aux toilettes, la préparation des cartables, de sandwichs pour l'école, sans oublier les caprices.

L'exemple suivant, extrait de Théra de Zeruya Shalev ${ }^{17}$, illustre parfaitement la démarche de ces écrivaines qui livrent les scènes quotidiennes dans leur intégralité en reconstituant tous les détails de l'épisode ainsi que l'ensemble des répliques ${ }^{18}$ :

Qu'est-ce que je te mets dans ton sandwich, je lui demande, mais il rouspète, j'en ai marre que tu me poses cette question, c'est la question la plus énervante du monde, et moi j'essaie de parlementer, qu'a-t-elle de si énervant, je veux juste que tu me dises ce que tu préfères, du fromage, du beurre de cacahuète, du chocolat? Tu passes ton temps à m'énerver exprès, commence-t-il carrément à hurler, pourquoi tu me cherches tout le temps, pourquoi tu ne veux que m'énerver. Guili, tu te trompes, je ne cherche pas à t'énerver, je te demande seulement ce que tu préfêres comme sandwich, je m'en fiche, marmonne-t-il, mets ce que tu veux, mais au moment où nous retrouvons devant la porte, il rouvre son cartable, en tire le sachet avec le sandwich et le jette par terre, je veux du chocolat pas du fromage, me reproche-t-il, alors je prépare à la hâte un nouveau sandwich en sachant que son sort sera similaire, jusqu'au jour où je décide de renoncer à la question et je lui prépare systématiquement deux sandwichs afin d'éviter un conflit qui, de toute façon, et quoi que je fasse, n'est jamais bien loin.

Lorsque le quotidien se constitue autour de la relation conjugale, le départ du mari modifie l'espace familier et rend désirable l'ensemble des activités domestiques qui symbolisent dorénavant le foyer et la vie commune. Vu de l'extérieur, le bonheur des autres se cristallise autant dans les éclats de rire que dans l'odeur des plats ou le cliquètement des fourchettes et des assiettes, qui s'échappent des fenêtres ou envahissent la cage d'escalier ${ }^{19}$.

Dans Mari et femme, l'héroïne de Zeruya Shalev éprouve un "besoin frénétique » de réintégrer son « train-train quotidien » pour «s'y recroqueviller comme un chien dans sa niche par une nuit d'orage $»^{20}$ (p. 93). Consciente de la banalité du paradis perdu, l'héroïne de Pliée de Tamar Gelbetz, exprime pourtant les mêmes regrets. Le regard nostalgique transfigure la "chronique ordinaire", parfaitement familière et même ennuyeuse d'un repas familial pris à la cuisine ${ }^{21}$ (p. 146).

\section{L'espace miroir}

Matériau romanesque à part entière, digne d'une description minutieuse, l'univers domestique acquiert aussi au fil de la narration la fonction d'un espace réflecteur où s'inscrivent les principaux événements de l'intrigue et les bouleversements psychologiques vécus par les personnages.

Dans les récits conçus autour d'une rupture (z. Shalev, S. Horn ou Tamar Gelbetz), le départ du mari, son retour (souvent provisoire) ainsi que la naissance d'une nouvelle aventure amoureuse se répercutent dans cet espace que représente la réalité quotidienne. Chez certaines écrivaines, les phases d'abattement qui suivent la rupture se traduisent par un refus de l'héroïne d'assumer les fonctions ménagères : les lits sont défaits, la vaisselle s'accumule dans l'évier, les restes de nourriture moisissent dans le réfrigérateur qui se vide ${ }^{22}$. Chez d'autres, l'absence du conjoint se traduit bien au contraire par un ordre excessif, la vraie vie, la vie de famille, étant synonyme d'un joyeux désordre. Vidé de son essence, l'appartement familial ressemble alors à une ville fantôme. La narratrice de Tamar Gelbetz énumère les pièces l'une après l'autre pour établir un nouvel état des lieux : la cuisine, autrefois " un volcan en éruption", se transforme en amas de cendres noires inertes et figées, la salle de bain, où personne ne 
se douche ne comporte plus aucune trace d'humidité, le salon où s'accumulaient jouets, télécommandes, livres, journaux, et miettes de pain est propre, mais désert (p. 14-15).

Hantée par un sentiment d'abandon, l'héroïne de Mari et femme perçoit le changement « dans un appartement dont le quotidien a changé du tout au tout, et où, semble-t-il, jamais pied masculin n'a pénétré.»(p.305). Et c'est devant la cuvette béante des toilettes, peut-être plus qu'ailleurs, qu'elle éprouve l'humiliation d'une femme délaissée (p. 271).

24 Le retour provisoire de l'homme, tout comme son départ, bouleverse l'espace quotidien, car il s'accompagne d'une intense activité domestique où l'héroïne retrouve ses « instincts de femme au foyer ${ }^{23}$ désireuse de recréer, grâce aux tâches ménagères qu'elle accomplit joyeusement, l'atmosphère d'autrefois, concoctant parfois un plat destiné à séduire ou à réconcilier ${ }^{24}$. Tamar Gelbetz décrit ces travaux longuement et avec délectation, livrant aux lecteurs la préparation minutieuse d'un plat qu'elle décrit étape par étape conformément à la recette ${ }^{25}$ (p. 65-68).

Destiné à refléter avant tout la relation entre l'homme et la femme, la mère et l'enfant, cet espace miroir sert aussi, plus rarement, à définir d'autres rapports, individuels ou collectifs. Dans Eden de Yaël Hedaya, les tâches ménagères compensent l'absence d'un véritable dialogue entre une mère vieillissante et sa fille. La multiplication de gestes quotidiens reflète, dans un rapprochement inversé, le vide affectif et l'absence de communication entre les deux personnages (pp. 296-297).

Chez Mira Magen, l'univers domestique permet aussi d'établir une comparaison entre religieux et laïques, car dans le récit féminin, la différence s'inscrit obligatoirement dans le quotidien, envahissant d'un côté, contrôlé de l'autre, vécu en commun ou individuellement, protégé derrière une porte close :

Les portes de Haya Horowitz et de Léah Grossman sont grand ouvertes. De deux côtés de l'escalier, leurs vies s'écoulent, entremêlées. Les bébés gigotent entre les jambes, rampent d'un appartement à l'autre [...].

Tous sont associés au chou-fleur dans la marmite, au dosage du paprika, au prix du lait basses calories et aux règles en retard de six jours.

Je ne suis pas très douée dans ce domaine, ma cuisine est médiocre, je n'ai pas de recettes surprenantes, ni pour le chou-fleur ni pour le brocoli, et mes règles tombent avec précision, de toute façon depuis six mois, je n'ai pas d'homme dans ma vie. ("Des Gerberas à moitié prix ", p. 12) 2 .

Bien plus qu'un simple arrière-plan, devenu objet de la narration et espace réflecteur, le quotidien dans cette prose acquiert rarement une valeur symbolique, sans doute en raison de son extension.

Vie amoureuse $e^{27}$, le premier volet de la trilogie de Zeruya Shalev, différent par son intrigue des romans évoqués précédemment, offre un exemple intéressant d'une tentative de symbolisation dans la présentation prémonitoire et parfois ironique des relations tumultueuses de l'héroïne avec les hommes. En passant de l'amant au mari, du mari à l'amant, la jeune femme établit une comparaison tantôt explicite tantôt implicite entre les deux hommes, leurs comportements, leurs performances amoureuses, mais aussi leur environnement quotidien avec un avantage marqué pour l'amant. Deux scènes qui se déroulent dans la cuisine semblent contredire ce constat, la menace d'un échec pesant autant sur l'un que sur l'autre. Le mari échouera sans doute à cause de l'univers domestique avec lequel il se confond (p.183). L'amant par manque d'engagement, autant sur le plan affectif que dans la vie de tous les jours (p. 158). 


\section{Et si on parlait de métaphores?} exploitant l'univers domestique dans la description métaphorique de faits, de gestes et de sentiments. Les nombreux comparants, issus du domaine culinaire ou inspirés par des tâches ménagères créent un contrepoint humoristique dans un monologue éploré d'une femme abandonnée par son mari. Les accents dramatiques que pourrait inspirer le récit détaillé des différentes phases de cette expérience sont contrebalancés par des éléments empruntés à la banalité quotidienne. Les travaux domestiques servent à décrire les disputes entre les conjoints ainsi que toute une gamme de sentiments, créant ainsi des métaphores inattendues : la colère de la femme est comparée à un fer à repasser surchauffé (p.118) ou à une eau de javel décapante (p.154); le sentiment d'angoisse à une poêle Tefal au revêtement particulièrement résistant, les pleurs ininterrompus à une fuite dans les toilettes et les infructueuses tentatives de trouver 
une consolation physique (en se masturbant) à l'effet que produit le Scotch bright sur la vaisselle (p. 126).

Les sucreries et les desserts sont particulièrement propices à l'évocation du bonheur : un seul baiser peut transformer la femme déprimée en crème vanille nappée de caramel, en fraises parsemées de morceaux de biscuits mélangés à la crème fraîche (p. 28). Ces descriptions se distinguent généralement par un déséquilibre dans la construction des figures, car un seul comparé génère de nombreux comparants :

Je suis une tête de choco collante, chocolat à l'extérieur, mousse blanche à l'intérieur [...]. Il me boit en une seconde sans paille me manipule m'aplatit et me déroule sous un rouleau à pâtisserie [...] il me fait monter comme un gâteau au fromage [...]. Je monte et m'épanouis enfournée dans son corps, et la merveilleuse douceur dégage une délicieuse odeur de levure qui se répand dans toute la maison (p. 117).

\section{Conclusion}

Dénoncer les pièges de la banalité quotidienne dans une démarche ironique ou évoquer sans amertume un univers domestique comme partie intégrante d'un vécu digne de narration, telles sont les deux principales tendances de cette écriture romanesque conjuguée au féminin. Deux options qui impliquent deux poétiques différentes, l'une déstructurante, l'autre conciliante, dénuées toutes deux de la tonalité dramatique des écrits féministes. Tandis que Orly Castel-Bloom tourne en dérision le sentiment maternel, le bonheur familial et l'amour romantique, Zeruya Shalev, Yaël Hedaya, Shifra Horn, Mira Magen et bien d'autres semblent y croire, en racontant un quotidien débarrassé de sa grisaille. Leurs héroïnes n'éprouvent ni ennui, ni mélancolie, ni désespoir. Aussi éloignées d'Anna Karenine que de Madame Bovary, ces femmes vivent leurs amours et leurs désamours au jour le jour sans jamais opposer l'élan romantique à la banalité quotidienne.

Au-delà de ces considérations thématiques, on rappellera le choix poétique de ces écrivaines qui accordent une large part à la description de faits et gestes anodins, considérés souvent comme un rituel. En favorisant la transmission minutieuse de scènes de la vie quotidienne et non leur résumé, elles diminuent l'impact des éléments dramatiques et les inscrivent non seulement dans l'espace, mais aussi dans la durée. Inspiré du vécu féminin, le figuratif confirme par le choix des comparants l'importance des thèmes abordés dans la narration.

\section{BIBLIOGRAPHIE}

\section{Cuvres citées}

ADLER, Malka (2001),בואי דודה נרקוד (Viens ma tante, on danse), Yediot Aharonot, Tel-Aviv.

AINI, Léa (1997), הרדופים (Oléandres), Ha-kibbutz ha-meuhad, Tel-Aviv.

CASTEL-BLOOM, Orly (1993), Dolly city, traduit par Rosie Pinhas-Delpeuch, Actes Sud, Arles. 
CASTEL-BLOOM, Orly (1995), Où je suis, traduit par Rosie Pinhas-Delpeuch, Actes Sud, Arles.

CASTEL-BLOOM, Orly (1998), La Mina Lisa, traduit par Rosie Pinhas-Delpeuch, Actes Sud, Arles.

GELBETZ, Tamar (2006), מקופלת (Pliée), Hargol/'Am 'Oved, Tel-Aviv.

HEDAYA, Yaël (2006), עדן (Eden), 'Am ‘Oved, Tel-Aviv.

KAHANA-CARMON, Amalia (1971), וירח בעמק אילון(Et la lune dans la vallée d'Ayalon), Ha-kibbutz ha-meuhad, Tel-Aviv.

KAHANA-CARMON, Amalia (1996), "לב הקיץ, לב האור 》 ("Au coeur de l'été, au cour de la

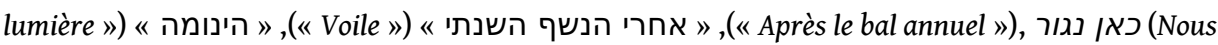
habiterons ici), (pp. 65-131, p. 221-240, p. 241-273).

MAGEN, Mira (1994), כפתורים רכוסים היטב (Des boutons bien boutonnés), Keter, Jérusalem.

MAGEN, Mira (2000), בשוכבי ובקומי, אישה (En me couchant, en me levant, femme), Keter, Jérusalem. ROZOVSKY, Miri (2001), כל הדרך הביתה (Tout le chemin du retour), 'Am 'Oved, Tel-Aviv.

SHALEV, Zeruya (2000), Vie amoureuse, traduit par Sylvie Cohen, Gallimard, Paris.

SHALEV, Zeruya (2002), Mari et femme, traduit par Laurence Sendrowicz, Gallimard, Paris.

SHALEV, Zeruya (2007), Théra, traduit par Laurence Sendrowicz, Gallimard, Paris.

SHEMER, Idit (2003), אז מה את באמת רוצה(Alors que veux-tu vraiment), Keter, Jérusalem.

\section{Critique et analyse littéraire}

ANSERSON, Linda (1990), Plotting Change: Contemporary Women's Fiction, Edward Arnold, London. BEN-SHAHAR, Rina (2003), « חבל על הטרנד : הפואטיקה של אורלי קסטל-בלום ( Tant pis pour le trand : la poétique de Orly Castel-Bloom »), לאיל"ש למלי (À l'occasion des 30 ans de l'association israélienne de linguistique appliquée), Tziv’onim, Jérusalem, p. 78-93.

COHEN, Tova (2007), קול עלמה עבריה(La Voix d'une femme juive), 'Am ‘Oved, Tel-Aviv. GREEN, Gayle (1979), Changing the Story, Indiana University press, Boomington and Indianapolis. RATOK, Lily (1994), קול אחר (Une autre voix), Ha-kibbutz ha-meuhad, Tel-Aviv.

SHAKED, Gershon (1998), 1980-1880 (La Prose hébraïque 1880-1998, vol.5), Keter/ Ha-kibbutz ha-meuhad, Jérusalem.

\section{NOTES}

1. Les titres originaux des œuvres non traduites sont indiqués dans la bibliographie. Cf. Tova Cohen, 2007.

2. Les titres de ses articles témoignent de son esprit belliqueux : « La femme de Brenner repart au galop », Moznaïm 69, 4, octobre 1985 ; "Elle n'écrit pas mal, mais seulement sur ce qui est marginal », Yedioht Ahronoht, 4.2.1988.

3. Ces dominantes thématiques s'accompagnent d'un changement de perspective. Parmi les nombreux écrits théoriques consacrés à ce sujet, on mentionnera: Gayle Green, 1979, Linda Anderson, 1990.

4. Amalia Kahana-Carmon, 1971.

5. Cf. l'analyse de Lilly Rattok 1994, p. 293.

6. Pour cette double vie, à la manière de Madame Bovary, voir Gershon Shaked, 1998, p. 311 et p. 316. 
7. Amalia Kahana-Carmon, 1996, pp. 241-273 [1 $1^{\text {re }}$ publication, 1984].

8. Ibid. respectivement, pp. 221-240 et p. 65-131 [1 $1^{\text {re }}$ publication 1977 et 1966].

9. Voir notamment les nouvelles de Léa Aïni, 1997, et de Miri Rozovsky, 2001.

10. Orly Castel-Bloom, 1998.

11. Pour la poétique de Orly Castel-Bloom, cf. Rina Ben-Shahar, 2003.

12. Orly Castel-Bloom, 1993.

13. Orly Castel-Bloom 1995.

14. Pour les romans étudiés cf. bibliographie ci-jointe.

15. Le terme « rituel » est employé par Zeruya Shalev, 2007, p. 117 et par Shiffra Horn, 2004, p. 34.

16. Voir également Zeruya Shalev 2007, Shiffra Horn, 2004 et Mira Magen, 2000.

17. Zeruya Shalev 2007.

18. Voir des scènes analogues dans : Mira Magen 2000, pp. 16-27 et Yaël Hedaya, 2006, pp. 46-48.

19. Cf. Zeruya Shalev, 2002, p. 211 et 2007. p. 83, p. 16; Tamar Gelbetz, 2006, p. 62.

20. Zeruya Shalev, 2002.

21. Gelbetz, 2006.

22. Cf. Shifra Horn, 2004, p. 318; Zeruya Shalev, 2007, p. 160 et 2002.

23. Cf. Tamar Gebeltz, 2006, p. 65.

24. Cf. Zeruya Shalev, 2007, p. 132, Tamar Gebeltz, ibid, pp. 65-68.

25. La longue description des préparatifs souligne l'échec de la séduction. Mais contrairement à Orly Castel-Bloom, l'humour ne mène pas à la dérision. Cf. également Zeruya Shalev 2002, pp. 60, 159.

26. Mira Magen, 1994.

27. Zeruya Shalev, 2000.

28.

La prose et non la poésie, puisque dans la poésie, ce type d'associations est à la fois plus fréquent et plus complexe.

29. Respectivement: Idith Shemer, 2003, p. 11, Malka Adler 2001, p. 184.

30.

On notera, au passage, les changements opérés dans l'écriture de cette romancière, marquée de plus en plus par la présence d'éléments figuratifs, surtout des comparaisons.

\section{RÉSUMÉS}

Les personnages féminins dans la littérature israélienne. Rejetée par les écrivains féministes tels qu'Amalia Cahana Karmon, considérée comme source d'insatisfaction, la vie d'intérieur devient la plupart du temps une facette indispensable de la trame des romans contemporains écrits par des auteurs israéliens tels que Zeruya, Yael Hedaya, Shifra Horn, Mira Magen, Tamar Gelbetz, etc. Une lecture minutieuse révèle des caractéristiques similaires dans le contenu fictionnel et les techniques narratives. Souvent, la trame se concentre sur une héroïne vivant la fin ou le début d'une histoire d'amour. Tout en explorant les différents stades de l'expérience sentimentale, l'auteur s'évertue également à décrire la relation quotidienne d'une mère et de son enfant. Étant un élément important du récit, la vie d'intérieur inspire à la fois des choix poétiques et stylistiques, particulièrement intéressants dans la construction de la métaphore traitant de la passion. 
Seuls quelques auteurs semblent se soucier de l'héritage d'Amalia Cahana Karmon. Parmi eux, Orly Castel Bloom dont les trames déconstruites et les personnages désarticulés ne laissent pas de place à la vie ordinaire ou à ses tâches ingrates.

Rejected by feminist writers as Amalia Cahana Karmon, considered like source of unhappiness, domestic life becomes most frequently an inseparate part of the plot in nowadays novels written by Israeli authors as Zeruya Shalev, Yael Hedaya, Shifra Horn, Mira Magen, Tamar Gelbetz, etc. A close reading reveals similar features in the choice of fictional materials and narrative techniques. Often, plot focuses on a heroine living the end or the beginning of a "love story". While exploring different stages of this sentimental experience, the writer describes also the daily relationship between mother and child. Being part of the story, domestic life inspires as well poetic and stylistic choice, particularly interesting in metaphor's construction dealing with passion.

Only few voices seem to carry on Amalia Cahana Karmon's legacy. Among them, Orly Castel Bloom who's deconstructed plot and disarticulated characters do not leave any place for ordinary life and its unrewarding tasks.

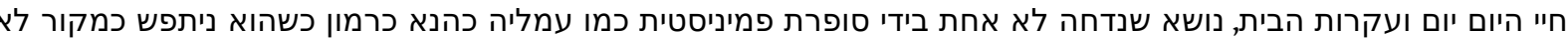

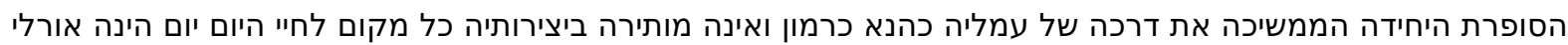

\section{INDEX}

Mots-clés : Kahana-Carmon Amalia (1926-), Castel-Bloom Orly (1960-), femmes dans la littérature, féminisme, quête identitaire, société israélienne

Index chronologique : vingtième siècle

Index géographique : Israël

\section{מילות מפתח}

שים בספרות, פמיניזם, החיפוש אחר הזהות, החברה הישראלית, ספרות, המאה: העשרים, עמליה כהנא כרמון, אורלי קסטל בלום

Thèmes : littérature

Keywords : literature, twentieth century, Women in literature, feminism, quest for identity, Israeli society, Kahana-Carmon Amalia (1926-), Castel-Bloom Orly (1960-), Israel 